Proceedings of Tools for A -90 .

Washington D.C., November 1990.

\title{
Generation of Feature Detectors for Texture Discrimination by Genetic Search.
}

\author{
J. W. Bala K. De Jong \\ Center for Artificial Intelligence \\ George Mason University \\ Fairfax, VA
}

\begin{abstract}
The paper presents a genetic algorithm based methodology for learning a set of feature detectors for texture discrimination. This methodology is implemented into a vision system that learns to classify noisy examples of different rexture classes by evolving populations of simple and texture-specific local spatial feature detectors.
\end{abstract}

\section{Introduction}

Texture provides very useful information for performing automatic interpretation and recognition of images by computer. Textural features can be crucial for the segmentation of an image and can serve as the basis for classifying image parts. Usually texture models are simulated and studied under the categories of pixel-based and region-based models. The texture recognition problem combines two troublesome characteristics. First, texture classes have prototypes which correlate highly with prototypes of different texture classes. Second, the texture examples (to be classified) are randomly distorted and noisy. Texture classification has been the focus of interest for a long time. Various investigations in pattern recognition have attempted to classify texture primarily at the image level. A number of approaches to texture analysis and classification problem have been developed over the years. Two fundamentally different approaches to texture analysis are the statistical approach and the structural approach. The statistical approach generates parameters to characterize the stochastic properties of the spatial distribution of gray levels in an image. The structural approach analyzes visual scenes in terms of the organization and relationships among its substructures.

This paper describes a novel variation of the statistical approach. Our strategy for selecting useful texture features involves the use of an adaptive search technique, a genetic algorithm, to efficiently search the space of spatial feature detectors for a high performance feature set.

\section{The Feature Selection Problem}

Any object or pattern which can be recognized and classified possesses a number of discriminatory properties or features. The first step in any recognition process, performed either by a machine or by a human being, is to choose discriminatory features and to measure these features. Feature selection in pattern recognition concerns the extraction of characteristic features or aturibures from received input data and the reduction of the dimensionality of pattern vectors. This is often referred to as the preprocessing and feature extraction problem.

It is evident that the number of features needed to successfully perform a given recognition task depends on the discriminatory qualities of the chosen features. However, the problem of feature selection is usually complicated by the fact that the most important features are either not easily measurable or, in many cases, their measurement is inhibited by economic considerations. The selection of an appropriate set of features, which support an acceptable performance in spite of difficulties encourtered in the extraction and selection process, is one of the most difficult tasks in the design of pattern recognition systern.

Feature selection may be accomplished by using two different approaches. The first one selects features independently of the performance of the classification scheme. Such an approach may be referred to as absolute fearre selection. An alternative approach is performancedependent feature selection, and its effectiveness is directly related to the performance of the classification system, usually in terms of the probability of correct recognition. This second approach of feabure selection is addressed in this project.

The inclusion of the feanure seloction and extraction stage effectively partitions the pattern recognition problem into iwo subproblems, as illustrated in Figure 1. 


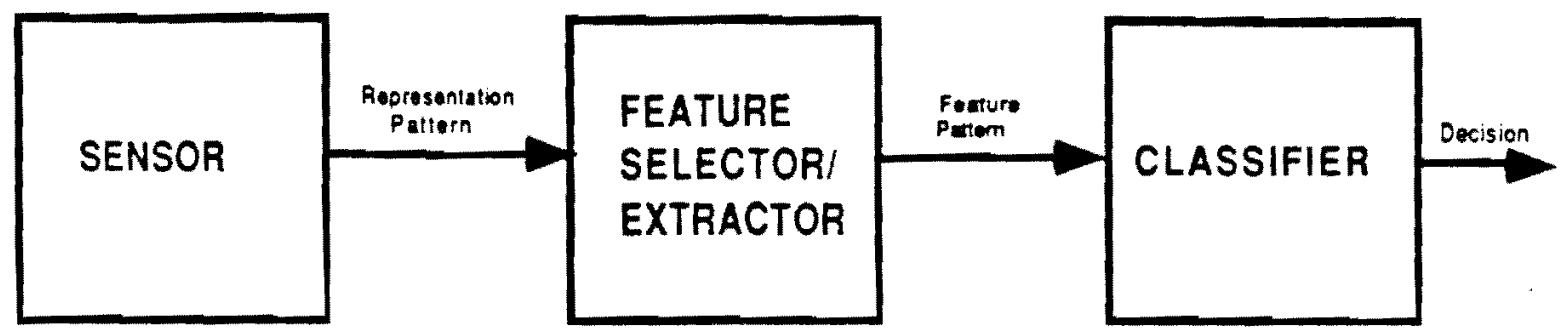

Figure 1. Partitioning of a pattem recognition problem by inclusion of a feature selection and extraction stage

\section{Feature Generation by Genetic Search}

A genetic algorithm [2] maintains a constant-sized population of candidate solutions, known as individuals. The initial seed population can be chosen randomly or on the basis of heuristics, if available for a given application. At each iteration, known as a generation, each individual is evaluated and recombined with others on the basis of its overall quality or fitness. The expected number of times an individual is selected for recombination is proportional to its fitness relative to the rest of population. The power of a genetic algorithm lies in its ability to exploit, in a highly efficient manner, information about a large number of individuals. By allocating more reproductive occurrences to above average individuals, the overall affect is an increase of the population's average fitness. New individuals are created using two main genetic recombination operators known as crossover and mutation. Crossover operates by selecting a random location in the genetic string of the parents (crossover point) and concatenating the initial segments of one parent with the final segment parent to create a new child. A second child is simultaneously generated using the remaining segments of the two parents. Mutation provides for occasional disturbance in the crossover operation by inverting one or more genetic elements during reproduction. This operation insures diversity in the genetic strings over long periods of time and prevents stagnation in the convergence of the optimization technique. The individuals in the population are typically represented using a binary notation to promote efficiency and application independence in the genetic operations.
We use genetic methods to generate the population of feature detectors. By using simple genetic operators (crossover, mutation, and selection), the population evolves and only the strongest elements (features) survive, thus contributing to the overall performance. Each detector contributes to textural class recognition and/or clustering. This contribution is used as an objective function to drive the machine learning procedures in its search for new, and useful feature detectors. Our method is illustrated in Figure 2.

\section{Binary String Coding and Genetic Operators}

Many possibilities exist for computing features to recognize texwures. The best features will be those which define an attribute space which most easily lends itself to partitioning by the classification algorithm. We use neighboring gray-level values as the feature attributes. These attributes are the simplest atrributes to characterize textures and the coding we use enables easy "manipulation" of this fearures by a genetic algorithm.

We use an $N^{*} N$ array centered on each pixel to define the neighborhood of interest. Each of the $\mathrm{N}^{*} \mathrm{~N}-1$ elements of the array are either zero or one, indicating which of the neighboring pixels are to be used to construct a feature vector. Figure 4 is an example of a $5 * 5$ array which extracts gray level values from 24 neighboring pixels. By selecting a variety of sample points in a texure area, a set of "typical" feature vectors can be extracted. For example, the following table illustrates feature vector values (10 gray levels resolution) extracted from seven

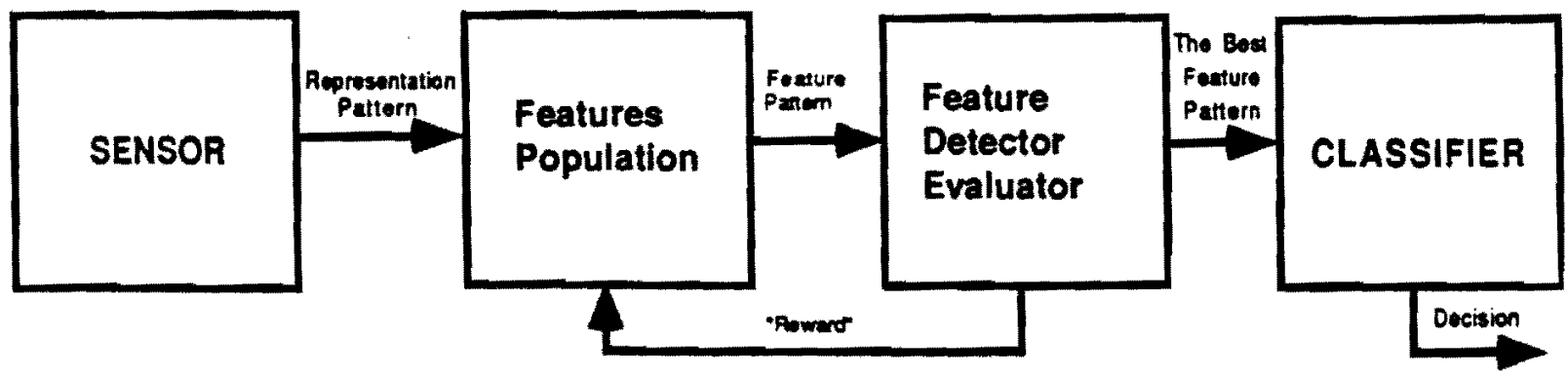

Figure 2. Genetic Algorithms approach to feature selection and extraction 
different positions in a textural area using the above described 5 by 5 detector.

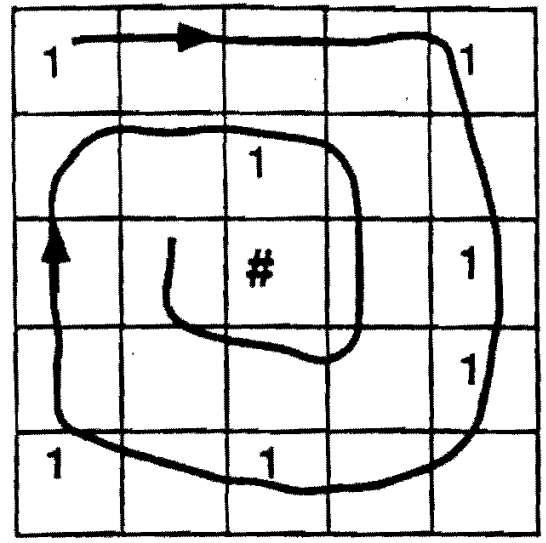

Figure 4. Feature coding for texture discrimination

Pixel positions

\begin{tabular}{lllllllll}
\multicolumn{10}{c}{ Sample Points } \\
\multicolumn{11}{c}{$\times 1$} & $\times 2$ & $\times 3$ & $\times 4$ & $\times 5$ & $\times 6$ & $\times 7$ & $\times 8$ \\
1 & 1 & 4 & 8 & 5 & 8 & 4 & 4 & 8 \\
2 & 1 & 8 & 6 & 7 & 4 & 6 & 3 & 8 \\
3 & 4 & 7 & 4 & 5 & 6 & 6 & 3 & 2 \\
4 & 3 & 6 & 5 & 5 & 4 & 3 & 2 & 8 \\
5 & 6 & 6 & 5 & 7 & 4 & 9 & 6 & 5 \\
6 & 5 & 4 & 3 & 6 & 8 & 4 & 3 & 5 \\
7 & 9 & 7 & 5 & 0 & 5 & 9 & 4 & 4
\end{tabular}

Selecting useful feature extraction templates of the type shown in Figure 4 is a different task. In order to use the genetic algorithms to search this space, it must be represented as a binary string. The spiral inside the template in Figure 4 represents the way we traverse the template's position to obtain a binary string representation for use with the genetic algorithm, resuluing in a binary string of

\section{"100010110010101001000000".}

Less significant bits of the extracted string (left side of the string) represent pixels located further from the centered pixel. More significant bits (right side of the string) represent close neighboring pixels to the center pixel. This coding method is very important in our algorithm. It reflects the way we describe textures, as statistical processes between neighboring pixels. The further the distances between the centered pixel and others in the window, the smaller the dependencies between pixels gray levels. By using this coding technique we are able to exchange important spatial discriminatory structures between different detectors in the population.

Figure 5 represents the idea of the crossover operation between two 5 by 5 detectors. The black dots in Figure 5 correspond to $1 \mathrm{~s}$ in the encoded strings depicted beneath detectors. Because of the spiral coding for the elements of population the detector windor is divided in to two regions according to the position of crossover point. The inner part (core) represents the coding of extraction information for the neighborhood of the central pixel. The other pan is represented by the left hand side position in the encoded string. The crossover operation, when applied to two detectors produces offspring detectors. By dividing the detector's area into regions closer and further to the central pixel, the information about relevant extraction discriminatory patterns for given texture classes can be easily transferred into subsequent generations.

\section{Parents detectors}

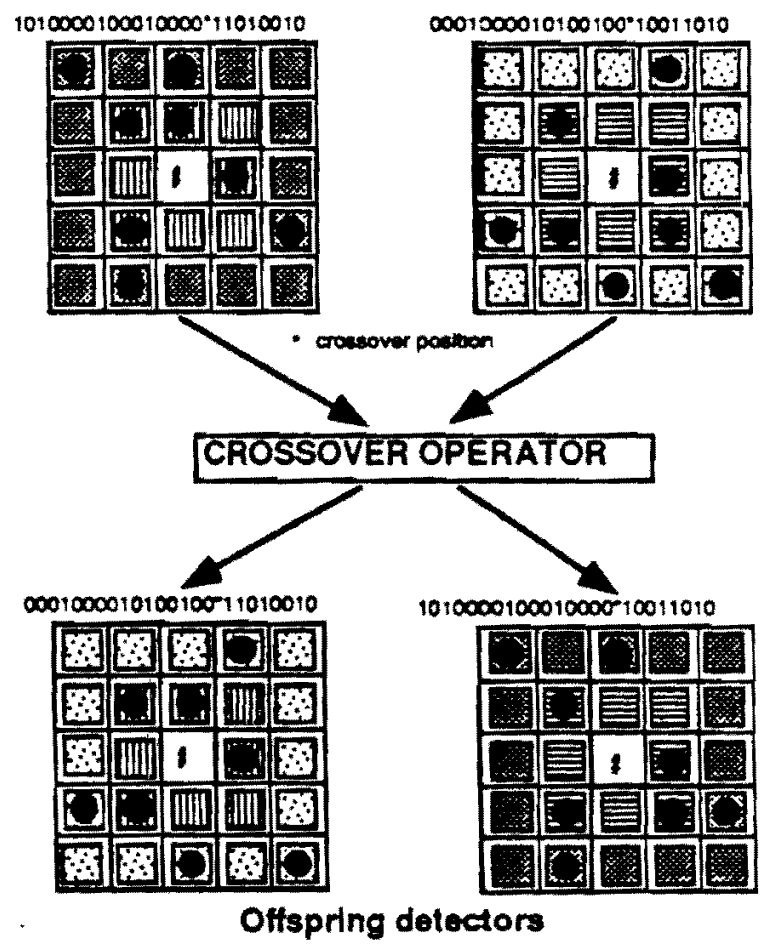

Figure 5. Crossover operation

The mutation operation in the initial phase of the system run is different than the standard one. Because our performance measure is the Euclidean distance (next section), the more 1s we have in the string, the more attributes the feature vector has, and the greater the distance between two vectors. This situation may cause instability in the system, where feature detectors with more attributes may dominate in the population. That is especially important during initial generations of the population. To aleviate this, the initial mutation operation (start-up mutation) is performed by shifting is in the feature string randomly to the left or to the right. This type of operation preserves the extracted vector length (the cotal number of positions with is in the string is the same). After a few initial generations, the 
system is already tuned up to its input data (textures) so that the standard mutation can be introduced.

\section{Feature Detector Performance Evaluation}

The evaluation of the feature detector is based on its ability to "cluster" members of training examples. For each example of a given training set, the Euclidean distance to examples of other classes is computed. Pattern classification techniques based on distance functions are one of the earliest concepts in automatic pattem recognition.

This method can yields practical and satisfactory results when the pattern classes tend to have clustering properies. We have decided to use it for two reasons. First, it is less computationally expensive than other methods which is important for a genetic search algorithm where performance measure for each detector in the population is computed in each cycle of the population. Second, even if we are unable to correctly classify examples from the training sets for a given feature extraction method, we can still compare the performance of the detectors and assign an evaluation value.

Our measure of performance is the total similarity between classes which is represented as the sum of the similarity measures between each two classes. The similarity measure between two classes is the sum of the similarity measures between all possible different pairs of examples (one from the first class and the other from the second class). If the distance is below a predefined threshold value, these two examples are considered to be "close" and statistics for each of the two classes are updated.

The measure we use for two examples is the square of Euclidean distance between these two examples. This measure for two vectors (examples) $x$ and $y$ of dimensionality $D$ is denoted as:

$$
\|x-y\|=\sum_{i=1}^{D}(x-y)^{2}
$$

where $x_{k}$ and $y \mathbf{k}$ are $k$-th components of $D$ dimensional vectors $x$ and $y$.

Let us suppose that we have $\mathbf{N}$ classes, $\mathbf{M}^{\mathbf{i}}$ examples in each class $(i=1, \ldots, N)$. Let $S$ be the number of detectors in the population (population size), and $T$ be the threshold distance value. The performance measure $p(v)$ of the feature detector $v(v=1, \ldots, S)$ is represented by the following formula:

$$
p(v)=\sum_{-1}^{N=1} \sum_{k=1}^{N} \sum_{i=1}^{N} \sum_{b=1}^{N} d\left(e_{i}^{k}, e_{k}^{p}\right)
$$

$e_{i}^{:}\left(e^{b}\right)$ is the $a(b)$-th example from the $i(k)$-th class

$$
d\left(e_{i}^{a}, e_{k}^{b}\right)=\left\{\begin{array}{l}
1 \text { if }\left\|e_{i}^{a}-e_{k}^{b}\right\|<T \\
0 \text { if }\left\|e_{i}^{a}-e_{z}^{b}\right\| \geq T
\end{array}\right.
$$

The performance measure of the whole population $P$ is denoted as:

$$
\mathrm{P}=\sum_{v=1}^{s} \mathrm{p}(v) / \mathrm{S},
$$

which is an average performance value of the population of feature detectors.

\section{Experimental Framework}

The Figure 6 depicts the 9 by 9 detector that has been used in experiments. The total length of a string is 81 bit positions.

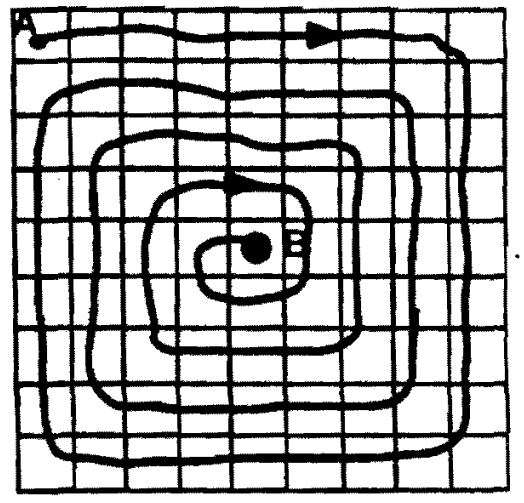

Figure 6. A large 9 by 9 window

By using the larger window, we are able to tune up our feature extraction process to different resolutions of lextures (windows 5 by 5 and 3 by 3 are subset of the 9 by 9 window). The point $A$ represents the left most position of the encoded string, and the point $B$ represents the right most position. The black dot is the central pixel, for which the feature detector is applied. The 9 by 9 window is scanned throughout all positions of a given textural area, and in each position an example of the class description is extracted with the number of attributes equal to the number of $1 \mathrm{~s}$ in the encoded string. Examples extracted from different pixel positions of the textural area represent its class description. The method was tested on 15 textural images from Brodatz 
[1] album of textures. The extraction area (leaming area) for each texture was 50 by 50 pixels. 500 locations were chosen randomly inside these areas to extract 500 feature vectors for each texture. The results of testing this methods on four specific textures are presented in the next section. These textures are depicted below in Figure 7.

\section{Experimental Results}

\subsection{Evolving Feature Sets}

Figure 8 shows the changes of similarity measure over 18 generations of the feature detectors population. This experiment was performed on the four textural images depicted in Figure 7. There were 35 individuals in the population. The total similarity measure (the sum of similarity measures of each class) drops from 1000 to 200 , thus yielding better clustering of these two classes. A similarity measure of 200 in the 18 th generation means that there are 200 pairs of extracted feature vectors from different classes ( 500 vectors in each class) at the distance below the threshold value. Similar results have been obtained for all different textural images.

Figure 9 shows the best detectors in the 15t, $6^{\text {th }}$ and the 18 th generation of population. These detectors show spatial arrangement of pixels positions (filled squares) that are important and relevant for discriminatory characteristics of these four textures. Although we use these detectors directly to extract atributes as the pixel gray levels values, it should be noticed that this higher level information obtained from these detectors (pixels structures) can be useful to "custom-design" standard extraction operators for specific needs (depending on input images). For example, we may choose a pecific mask operator from the collection of Law's mask operators. This aspect is the subject of further research.

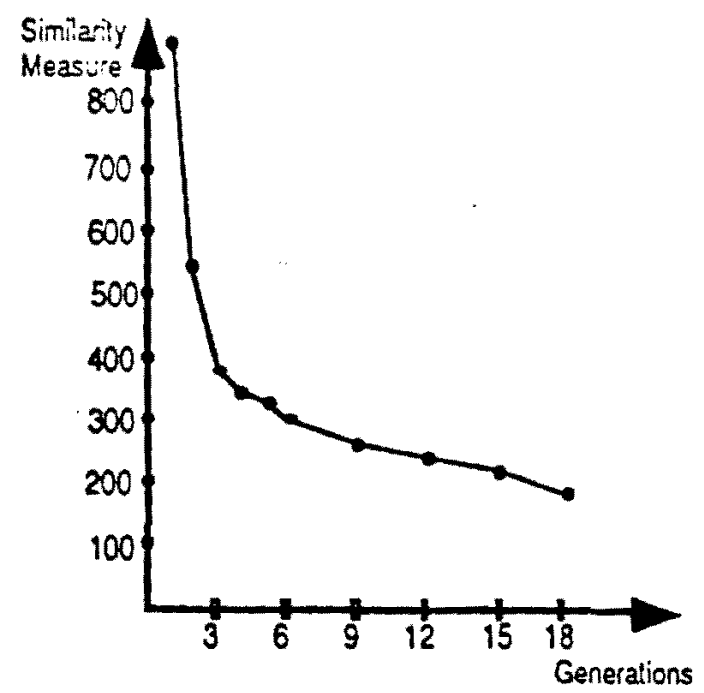

Figure 8. Total similarity measure for four textures

\subsection{Testing Clustering Degree}

We use the AQ leaming module to generate symbolic rule-based descriptions of the texture classes from the feature sets selected by the genetic algorithm. The AQ15 program is based on the $A Q$ algorithm [7], which generates decision rules from a set of examples. When building a decision rule, AQ performs a heuristic search through a space of logical expressions to determine those that account for all positive examples and no negative ones.
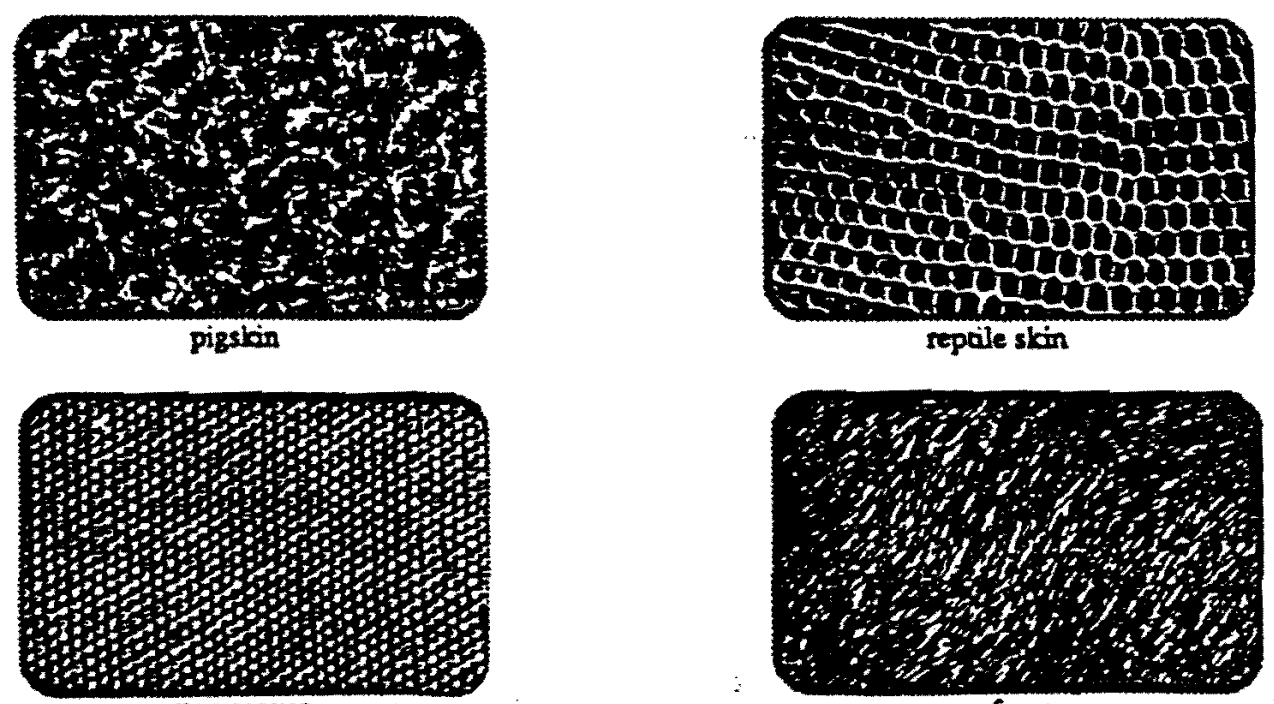

cotron carrves

$\therefore=-1$

Figure 7. Texaral images used in experiments 
Learning examples are given in the form of events, which are vectors of attribute values. Events from a The given class are considered its positive examples, and all other events are considered its negative examples. For each class a decision rule is produced that covers all positive examples and no negative ones.

A decision rule is a disjunction of complexes describing all positive examples and none of the negative examples of the concept. A complex is a conjunction of selectors. A cover is formed for each decision class separately. It defines the condition part of a corresponding decision rule. The following is an example of decision rule (with 4 complexes):

$$
\begin{aligned}
& \text { 1. }[x 1=5 . .9] \quad[x 3=6 . .9] \quad[x 9=0 . .15] \\
& \text { (total: } 10 \text {, unique 6) } \\
& \text { 2. }[x 4=6.99] \quad[x 7=6 . .9] \quad[x 9=0 . .16] \\
& \text { (total:8, urique } 5 \text { ) } \\
& \text { 3. }[x 2=4 . .9] \quad[x 4=4 . .9] \quad[\times 6=0 . .5] \\
& {[\times 9=0, .15]} \\
& \text { (total:8, unique 5) } \\
& \text { 4. }[x 3=5 . .9] \quad[x 5=0 . .6] \quad[x 6=0 . .6] \\
& {[x 9=0 . .16]} \\
& \text { (total: } 2 \text { unique } 1 \text { ) }
\end{aligned}
$$

The following example with nine attributes

$$
\langle 160464569\rangle
$$

is covered by the third complex of the above decision rale. We can notice that $\times 2=6$ is satisfied by the first selector of this complex, $x 4=4$ by the second selector, $x 6=4$ by the third, and $x 9=9$ by the last selector. Since there are no selectors for other attribute values $(x 1, x 3$, $x 5, x 7, x 8)$, they are satisfied by this complex.

For each complex the total number of examples covered by this complex and the number of unique examples covered only by this complex are presented. Feature sets which produce better clusters will result in simpler symbolic descriptions of the feature classes in the sense that fewer complexes are in the cover (more examples are covered by the first complexes of the class description). The following is the decision class with three complexes:

$$
\begin{aligned}
& \text { 1. } \begin{array}{l}
{[\times 2=2 . .4] \quad[x 3=10 \ldots 13] \quad[x 5=7 . .9]} \\
\text { (total:17, unique } 10)
\end{array} \\
& \text { 2. } \begin{array}{l}
{[\times 1=5 . .9] \quad[\times 2=6] \quad[x 7=0 . .11]} \\
\text { (total:12. unique } 7)
\end{array} \\
& \text { 3. } \\
& \begin{array}{l}
{[\times 2=4 . .9] \quad[x 4=4 . .9] \quad[x 6=0 . .5]} \\
\text { (total:5, unique 4) }
\end{array}
\end{aligned}
$$

It has less complexes than the previous description and each complex covers more examples. The third complex of this decision covers the same example ( $<1 \quad 6 \quad 046$ $\left.\begin{array}{llll}5 & 6 & 9 & >\end{array}\right)$.

During the second, the sixth, and the final generations of population, we select the best feature detector in the population to extract a set of feature vectors as to the $A Q$ module for classification. We observe a significant improvement in the class description generated by $A Q$ from one generation to the next. The number of complexes in the class cover drops by $12 \%$. Also the number of total and unique vectors covered by the first complex of a given class cover is about $8-10 \%$ higher than that for the first cover generated during the initial generation of the population. Different experiments performed on numerous images clearly show that successive generations of the population produce a better degree of clustering for a given class description.

\section{Conclusion}

Preliminary work with an application of genetic algorithms to a texture feature selection has been described. The results obtained confirm the utility of the genetic algorithm in solving problems within the specified domain. The next step would be to test usefulress of this genetic approach to more complex texture discrimination and general computer vision operators. More complex operators should be tested.

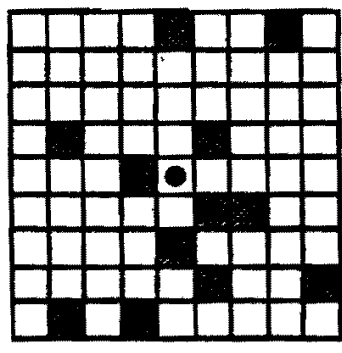

a

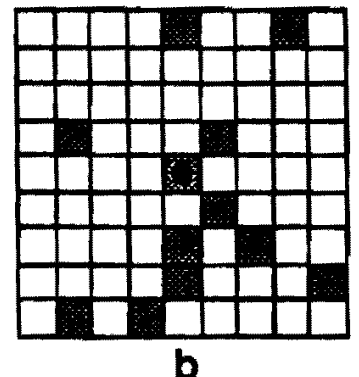

b

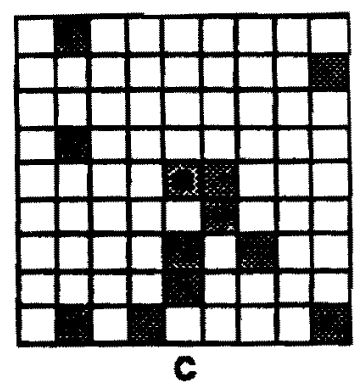

Figure 9. An example of the best detectors in (a) the 1st, (b) 6th, and (c) 18th generation of population 
For example, we may introduce weights to our previous operator. Three bits are reserved for each pixel position to code eight levels of weighting information. The total bit length for the suring is $9 \times 9 \times 3=243$. The string coding for such an operator is depicted in Figure 10.

\section{$" 010 \cdot 000 \cdot 101 \cdot 000 \cdot 000 \cdot 000 \cdot 000-000 \cdot 001 \ldots$.}

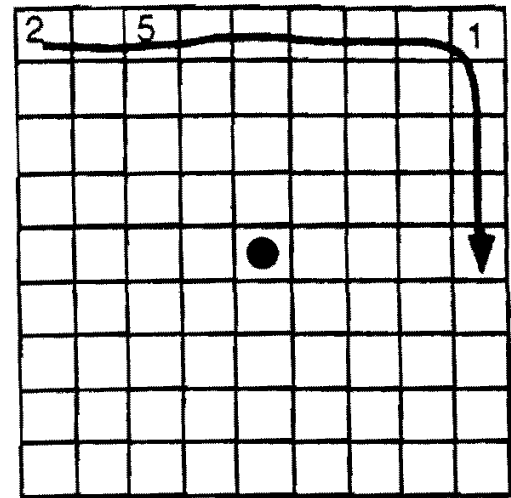

Figure 10. Coding with weights

There is another aspect of the genetic approach which can be exploited. Namely, this approach enables the exploration of different feature vectors. The final class representation can be generated by using a few of the best operators of the population. These operators can be used to generate several descriptions of the same class. This "multi-view" class representation encodes a more complete description of the class, that is of significant importance in the recognition phase of our system yielding better results than a single view representation of the class. This characteristic of the genetic approach to fearure extraction is the subject of further research.

\section{ACKNOWLEDGMENTS}

The authors wish to thank Dr Ryszard Michalski, Dr Gheorghe Tecuci, and Dr Harry Wechsler for valuable comments and discussion, and Janet Holmes for editing suggestions.

This research was done in the Arificial Intelligence Center of George Mason University. Research activities of the Center are sponsored in part by the Defense Advanced Research Projects Agency under grant, administrated by the Office of Naval Research, No. NOCO14-87-K-0874, in part by the Office of Naval Research under grant No. N00014-K0226, and in part by the Office of Naval Research under grant No. N00014-88-K0397.

\section{REFERENCES}

[1] Brodatz, P., : A Photographic Album for Arts and Design, Toronto, Dover Publishing Co., 1966.

[2] De Jong, K., : Learning with Genetic Algorithms: An Overview, Machine Learning 3; 123-138, Kluwer Academic Publishers, 1988.

[3] Fitzpaurick, J.M. and J.J. Grefenstette, : Genetic Algorithms in Noisy Environments, Machine Learning 3 pp. 101-12-(1988).

[5] Fitzpatrick, J.M., J.J. Grefenstette, and D. Van Gucht, : Image Registration by Genetic Search, Proceedings of IEEE Southeastem Conference, pp. 460 464 (1984).

[6] Gillies, A., : Machine Learning Procedures for Generating Image Domain Feature Detectors, Ph.D. dissertation, Computer and Communication Sciences in The University of Michigan, 1985.

[7] Michalski, R.S., Mozetic I., Hong J R., Lavrac N., :The $A Q$ Inductive Learning System, Report No. UIUCDCS-R-86-1260, Department of Computer Science, University of Illinois at Urbana-Champagne, July 1986.

[8] Stadnyk, I., : Schemata Recombination in Pattern Recognition Problems. Genetic algorithm and their applications: Proceedings of the Second Intemational Conference on Genetic Algorithms, pp. 27-35.

[9] Tou, J., Gonzalez, R. : Pattern Recognition Principle, Addinson-Wesley Publishing Company, 1974. 



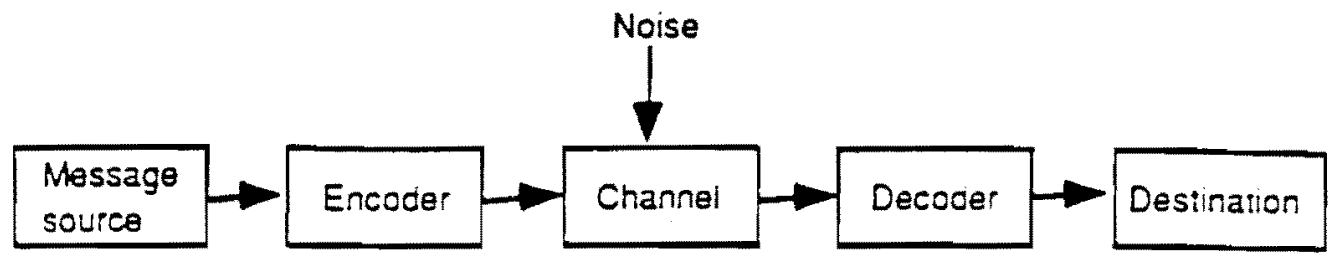

Figure 1: A typica message transmission path from source to destination

A variety of codes such as linear block codes, cyclic codes, burst error correcting codes. and convolutional codes are used for error correction [13]. In this paper we restrict our attention to block error correction codes which are charactenzed by the following parameters:

1) The Arity of the code (q). This represents the number of distinct symbols in the alphabet used in the code. Binary codes, one of the most common. have arity 2 and use the alphabet $[0,1)$.

2) The Length of a code (1). This is the number of leucts (from the alphabet) needed to make a word (vector) in the code, where a code denotes a set of words (vectors) which are used to transmit a message.

3) The Size of a code (M). The size of a code is the number of disunct words (vectors) in the code used to compose the messages to be sent

4) Distance (d) of a codc. The disunce of a code is the minimum Hamming disunce between pairs of words (vectors) belonging to the code. The Hamming disunce between two vectors $x$ and $y$, denoted by $d(x, y)$ is the number of positions in which the vectors $x$ and $y$ differ. In other words the Hamming distunce between $x$ and $y$ denotes the minimum number of substitution opernuons that must be performed to conver the vector $x$ : 0 the vector $y$. A code $C$ can correct up to $t$ errors if $d(C)>(2 i+1)$, where, $d(C)=\min (d(x, y) \mid x, y \in C$. $x \neq y)$.

A repetition code of length $n$ is a simple example of a block error correction code. It is defined for any arity; for a binary repeation code, each bit is repeated $n$ imes. An $n$ bit code toleraies floor $\left(\frac{n-1}{2}\right)$ bits of errors in each block of $\mathrm{n}$ bits. The information content in each block of $\mathrm{n}$ bits is 1 bic

An (n.M,d) code is a code of length n, contuining $M$ code words and having minimum disiance $d$. A good (n.M.d) code has a small n (renlecuing smaller redundancy and faster transmission), a large $M$ (denoung a larger vocabulary) and large d (indicaung greater tolennce to noise and error). Figure 2 lists theoreucal maximum values of $M$ (in some cases. esumation of lower and upper bounds of $M$ ) for some combinatuons of $n$ and $d$ (Source: $[10,15])$.

\begin{tabular}{|c|c|c|c|}
\hline$n$ & $d=3$ & $d=5$ & $d=7$ \\
\hline 5 & 4 & 2 & - \\
\hline 6 & 8 & 2 & - \\
\hline 7 & 16 & 2 & 2 \\
\hline 8 & 20 & 4 & 2 \\
\hline 9 & 40 & 6 & 2 \\
\hline 10 & 72.79 & 12 & 2 \\
\hline 11 & 144.158 & 24 & 4 \\
\hline 12 & 256 & 32 & 4 \\
\hline 13 & 512 & 64 & 8 \\
\hline 14 & 1024 & 128 & 16 \\
\hline 15 & $2(448$ & 256 & 32 \\
\hline 16 & 2560.3276 & 256.340 & 36.37 \\
\hline
\end{tabular}

Figure 2: Theoretical limit on the size for codes of vanous Ienghts and disunces

Although such bounds provide some insight into the best we can hope to achicve, there is frequently no guidance available as to how wo construct an actual code with (near) maximal distance. As the code size (n) increases, the search space of possible codes grows exponentially, ruling out any possibility of systematic or random search. For $(7,16,3)$ codes, the search space is at least $10^{20}$.

This paper describes experiments and results in using genetic algorithms as an efricient adaptive search stotegy for "discovering" maximal distance codes. Figure 3 shows an example of a (7.16.3) maxumal distance code solution. Solucions are in general not unique, but are sparse in the space to be searched.

\section{Representation Issues}

In order to apply genetic algorithms as an adaptive search stratesy, the problem of interest must be mapped into a representation suitable for genetic search. The simplest approach is to represent points in the space to be searched as ixed-length binary strings to which the standard geneuc operators of crossover and mutation can be applied when producing new oflspring [2]. For the coding domain. concatenation of the $M$ code words. each of length $n$ into one long binary sting. represents a suiuble mapping. Using this approach. a $(7,16.3)$ code would need a tola of 112 bits to represent 16 words (vectors) each of which 


\section{0 \\ 0001011 \\ 0010110 \\ 0011101 \\ 0100111 \\ 0101100 \\ 0110001 \\ 0111010 \\ 1000101 \\ 1001110 \\ 1010011 \\ .1011000 \\ 1100010 \\ 1101001 \\ 1110100 \\ 1111111}

Figure 3: A sample maximal distance code of length 7.

is 7 bits in length. Since we wish to maximize the minimum mutual distance among the words, it is clear that the performance of a code is dependent only on the collective composition of the words and is independent of the order in which the words in the code are represented. Soring the code in the decreasing order of the words reduces all equivalent codes to a single representation. In the case of a $(7,16.3)$ code, this heurisuc reduces both the seareh space and the number of possible solutuons by a factor of 16!. Initial experimenis showed that it is more profilable to discover a good code in the reduced space. One possible rezsons for this behavior is that it dramatucally reduces competition between potenual soluuons having high degree of bitwise dissimilanty, but idenucal evaluauon functions. Consequendy we adopted the sored represenvition for the expenments reported here.

\section{Evaluation Function}

A good evaluation function is vital to the successful applicauon of GAS to a problem. Evaluauon funcions provide goal-directed feedback sbout the fitness of individuals in the population. The adaouve behavior of the Gis deocnds on this feedback to drive the population towards better overall periormance. In this respect the objecuve lunction does not always consutute a good evaluation function.

Let $\boldsymbol{C}_{1} L, n$ ) Jenote the set of all possible codes of lengun $L$ and size n. Lut dix. $y$ ) denote Humming distance betiveen strings $x$ and $y$ of leneth $L$. Lut $J(C)=$ min|d(x. $y) \mid x . y \leqq C, x=y]$. Our goal is to discover

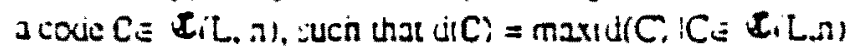
the minumum Jistance between unv pair of voros is hignest posstole. Our oblecure tuncion di Cl, wnten we visn to maximize maps all $\left(\begin{array}{l}a \\ a\end{array}\right)$ codes unto $L+1$ possible distances 10.2 . C.euriv 16 lacks joritiv to uscaminate xetiven promising ane run-rromising coues.
It is possible to remedy the situation by choosing an evaluation function $\mathrm{f}(\mathrm{C})$ such that it has many values in the co-domain, and if $\mathrm{d}(\mathrm{C})$ denotes $\max (\mathrm{d}(\mathrm{C})$ ) then $\mathrm{f}(\mathrm{C})$ denotes $\max (\mathrm{f}(\mathrm{C})$ ) over the space of all possible codes. $C(L, n)$. The objective function $d(C)$ is very sensitive to small changes in the codes. An evaluation function $f(C)$ may be chosen to provide a smoother gradient over bit changes in the codes. We used the following evaluation function initially: $f_{1}(C)=\frac{1}{\sum_{i=1}^{L} \min d_{i j} j=1 . .16 ; j \neq i}$ where $d_{i j}$ represents Hamming distance between words $i$ and $\mathrm{j}$ in the code $\mathrm{C}$.

However, the results of our initial experiments were discouraging and were partially attribuled to an inadequate choice of the evaluation function. The function $f_{1}(C)$ is better than $d(C)$, but still is not very helpful for GAs in terms of providing a good feedback. Consider two hypothetical codes $C_{1}$ and $C_{2}$, such that $C_{1}$ has the minimum mutual distance of 2.0 , and the average mumal distance of 2.5. and $\mathrm{C}_{2}$ has the minimum mutual disunce of 2.0, and the average mutual distance of 3.0. The code $C_{2}$ secms superior in terms of its proximity 10 opumal solution and therefore deserves a beuter fitness value. The evaluation function $f_{l}(C)$ is unable to discriminate between the two codes $C_{1}$ and $C_{2}$ and it is not effecuve in driving the population towards more desired code configurations. Note that a function which rewms average disunce between the pairs of vectors in the code is not $a$ good fitness function. It is easy to consuruct codes that maximize this critera, but have a minimum distance of 0 . For cxampic, a code consisting of 8 copies of all 1 vectors and 8 copies of all 0 vectors, has the average disunce of 3.5. which is the maximum for a 7 bit code of 16 size. but has a minimum mutual distance of 0 .

One way to think of the problem of finding a (7.16. $\left.d_{\text {max }}\right)$ code is to visualize placing 16 individuals on the comers of seven dimensional space so as to maximize the mutual distance between them. The situation is andogous to paructes of equal charge urying to position unemseives in the minimum energy configuration in a bounded space. The cvalusuion function

$$
\mathfrak{f}(\mathrm{C})=\sum_{i=1}^{L} \sum_{j=1 ; j \neq i}^{L} \frac{1}{d_{i j}^{2}}
$$

captures this idea and satisties all the desirable requirements such as being inderendent of the length or size of the code. Ind providing a smoother gradient with respect to the evaluation function. It resulted in atgnulicantiv better GA periormance and was used ist the expenments reported in this facer.

\section{Experimental Results}

In ull or the expenments reponed tere we used GEVEEIS (1.U ucsd). I version of GE.VESIS she!l uryenally

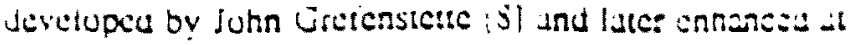


While they represent a significant improvement over andom search, none of the experiments converged to an opimal code in 1,000,000 cycles. The on-line and off-line performances were relatively stagnant for at least 600,000 cycles in each case, which indicated that convergence in the near future was unlikely.

\subsection{Changing the Alphabet Size}

There were a number of possible directions one could lake at this point. We were curious as to whether this might be an application in which treating individual code words as indivisible units (with respect to genetic operators) might improve things as is occasionally suggested (Antonisse, 1989) in spite of the theoretical results to the contrary (Holland. 1975). We defined a modified crossover operator in which crossover points could occur only at code word boundaries while the crossover rate remained at 0.6 . A code word was selected for mulation with probability of 0.01 . Once a word is selected, exactly one of the bits in the word was mulated at random.

With these changes, a set of experiments was run using the identical seeds and other parameters as the previous set of experiments. The modified GA discovered an optimal code in about 350,000 cycles on the average (four experiments with different seeds). Figure 6 shows codes discovered with each seed. we of the words in the code has a Hamming distance of at least 3 from its nearest neighbors. This results are surprising and contrary to the established belief that GA representauons using smaller sized alphabets are preferred over larger ones. Further analysis is required to undersund these resuls more fully.
Even though the evaluation function is multi-modal, in a given experiment the algorithm drives the population to one of the peaks, rather than dividing the population of different peaks. This suggests another direction for funure experiments: to study the effects of larger populations and the use of techniques to permit the evolution of "subspecies" around competing opumal peaks.

\subsection{Increasing the Number of Crossover Points.}

We were also curious if in this domain increasing the number of crossover points would have a significant effect on the ability to find optimal codes. The crossover operator was modified to perform 4 (rather than 2) point crossover. A set of four experiments was run in which the random number seeds. as well as all the parameters were kept idenucal to the previous experiments. Figure 7 summarizes the results. No significant changes were observed. Three of the populations converged to a desirable solution within 1,000,000 cycles and the other did not Time did not permit a more extensive analysis of the effocts of increasing the number of crossover points.

\section{5.t Scaling Up to Larger Coding Problems}

Anocher imporant issue is how well the performance of GAs scales up to larger problems. We were able to compare results on an $(8,16,4)$ problem which required a 128 bit representation (an increase of a factor of $2^{16}$. 65000 in the size of the search space).

\begin{tabular}{|c|c|c|c|c|c|}
\hline Expenment & 1 & 2 & 3 & \multicolumn{2}{|l|}{4} \\
\hline Crcle & $430.1 \times 10$ & $270.000)$ & 390.000 & 310.000 & \\
\hline Evaluauon functuon $f(C)$ & 19.6525 & 19.6525 & 19.6525 & 19.6525 & \\
\hline $\begin{array}{l}\text { Code words and Hamming } \\
\text { disunce from their nearest } \\
\text { reighbors }\end{array}$ & $\begin{array}{ll}1111011 & 3 \\
1110101 & 3 \\
1101110 & 3 \\
1100000 & 3 \\
1011000 & 3 \\
1010110 & 3 \\
1001101 & 3 \\
1000011 & 3 \\
0111100 & 3 \\
0110010 & 3 \\
0101001 & 3 \\
0100111 & 3 \\
00111111 & 3 \\
0010001 & 3 \\
0 x 011110 & \\
\text { (n) } & 31111\end{array}$ & $\begin{array}{ll}1111101 & 3 \\
1110011 & 3 \\
1101000 & 3 \\
1100110 & 3 \\
1011010 & 3 \\
1010100 & 3 \\
10011111 & 3 \\
1000001 & 3 \\
0111110 & 3 \\
0110000 & 3 \\
0101011 & 3 \\
0100101 & 3 \\
0011001 & 3 \\
0010111 & 3 \\
00011100 & 3 \\
1 & \end{array}$ & $\begin{array}{l}1111110 \\
1111001 \\
1100100 \\
1100011 \\
1010101 \\
1010010 \\
1001111 \\
1001000 \\
0110111 \\
0110000 \\
0101101 \\
0101010 \\
0011110 \\
0011011 \\
0000110 \\
\text { phruvil }\end{array}$ & $\begin{array}{l}1111010 \\
1110001 \\
1101101 \\
1100110 \\
1011100 \\
1010111 \\
1001011 \\
1000000 \\
0111111 \\
0110100 \\
0101000 \\
0100011 \\
0011001 \\
0010010 \\
0001110 \\
m \times 0 ! 0 !\end{array}$ & $\begin{array}{l}3 \\
3 \\
3 \\
3 \\
3 \\
3 \\
3 \\
3 \\
3 \\
3 \\
3 \\
3 \\
3 \\
3 \\
3 \\
3\end{array}$ \\
\hline
\end{tabular}




\begin{tabular}{|c|c|c|c|c|c|c|c|c|}
\hline Exnonment & \multicolumn{2}{|l|}{1} & \multicolumn{2}{|c|}{2} & \multicolumn{2}{|l|}{3} & \multicolumn{2}{|c|}{4} \\
\hline Crele & \multicolumn{2}{|c|}{240.000} & \multicolumn{2}{|c|}{210.000} & \multicolumn{2}{|c|}{290.000} & \multicolumn{2}{|c|}{600.000} \\
\hline Evaluztion funcuon $f_{2}(\mathrm{C})$ & \multicolumn{2}{|c|}{19.6525} & \multicolumn{2}{|c|}{20.450894} & \multicolumn{2}{|c|}{19.6525} & \multicolumn{2}{|c|}{19.6525} \\
\hline $\begin{array}{l}\text { Coas words and Hamming } \\
\text { distance from their nearest } \\
\text { ne:zhbors }\end{array}$ & $\begin{array}{l}1111100 \\
1110010 \\
1101001 \\
1100111 \\
1011011 \\
1010101 \\
1001110 \\
1000000 \\
0111111 \\
0110001 \\
0101010 \\
0100100 \\
0011000 \\
0010110 \\
0001101 \\
0000011\end{array}$ & $\begin{array}{l}3 \\
3 \\
3 \\
3 \\
3 \\
3 \\
3 \\
3 \\
3 \\
3 \\
3 \\
3 \\
3 \\
3 \\
3 \\
3\end{array}$ & $\begin{array}{l}1111111 \\
1110001 \\
1101000 \\
1100110 \\
1011100 \\
1011011 \\
1000101 \\
1000010 \\
0111010 \\
0110100 \\
0101101 \\
0100011 \\
0010111 \\
0010000 \\
0001110 \\
0001001\end{array}$ & $\begin{array}{l}2 \\
3 \\
3 \\
2 \\
3 \\
2 \\
3 \\
2 \\
3 \\
2 \\
2 \\
3 \\
3 \\
2 \\
3 \\
2\end{array}$ & $\begin{array}{l}1111000 \\
1110110 \\
1101111 \\
1100001 \\
1011101 \\
1010011 \\
1001010 \\
1000100 \\
0111011 \\
0110101 \\
0101100 \\
0100010 \\
0011110 \\
0010000 \\
0001001 \\
0000111\end{array}$ & $\begin{array}{l}3 \\
3 \\
3 \\
3 \\
3 \\
3 \\
3 \\
3 \\
3 \\
3 \\
3 \\
3 \\
3 \\
3 \\
3 \\
3\end{array}$ & $\begin{array}{l}1111000 \\
1110111 \\
1101011 \\
1100100 \\
1011101 \\
1010010 \\
1001110 \\
1000001 \\
0111110 \\
0110001 \\
0101101 \\
0100010 \\
0011011 \\
0010100 \\
0001000 \\
0000111\end{array}$ & $\begin{array}{l}3 \\
3 \\
3 \\
3 \\
3 \\
3 \\
3 \\
3 \\
3 \\
3 \\
3 \\
3 \\
3\end{array}$ \\
\hline
\end{tabular}

Figure 7: Examples of best codes found using four point crossover.

In this case we knew that distance 4 optimal codes existed from a theorem in coding theory which states that if $M$ is the largest value for a $(n, M, d)$ code where $d$ is odd. then there exists a $(n+1, M, d+1)$ eode, such that $M$ is also the largest value for a $(n+1, M, d+1)$ code. Since we already have opumal $(7,16,3)$ codes in hand, this assers the existence of a $(8,16,4)$ opumal code.

It is easy to construct such a code starting from a (7, 16,3). An additional bit is appended at the end of every 7 bit vector from $(7,16,3$ ) code such that the odd (or even) parity is preserved. This construction suggests that the number of solutions for $(8,16,4)$ code are about 16 imes the number of solutions for $(7,16,3)$ code. (For each solution in $(7,16,3)$ space we can generate two solutions by choosing either even or odd parity for the eighth bit. And additional factor of eight is cxplained by the freedom to insert the eighth bit on either side of one of the 7 bits.) The important observation here is that since, as noted above, the size of the search space has increased by a factor of $2^{16}$. the density of optimal solutions has decreased.

Figure 8 presents the results of two experiments on the $(8.16 .4)$ problem. Nouce that no combinatorial increase in convergence time is observed as the search space size increases combinatorially. This is a suprising result which needs to be furcher tested on larger coding problems.

\section{Conclusions and Future Research}

The iniual experiments presented here provide encounging results regarding the applieation of GAs to the discovery of communication codes. Clearly additional work needs to be done to clarify several important issues: the apparent advantage of a larger alphabet size in the GA represenution; the poicnual advaniage of a speciation mechanism; and effects on performance when scaling up wo larger problems with no known solutions.

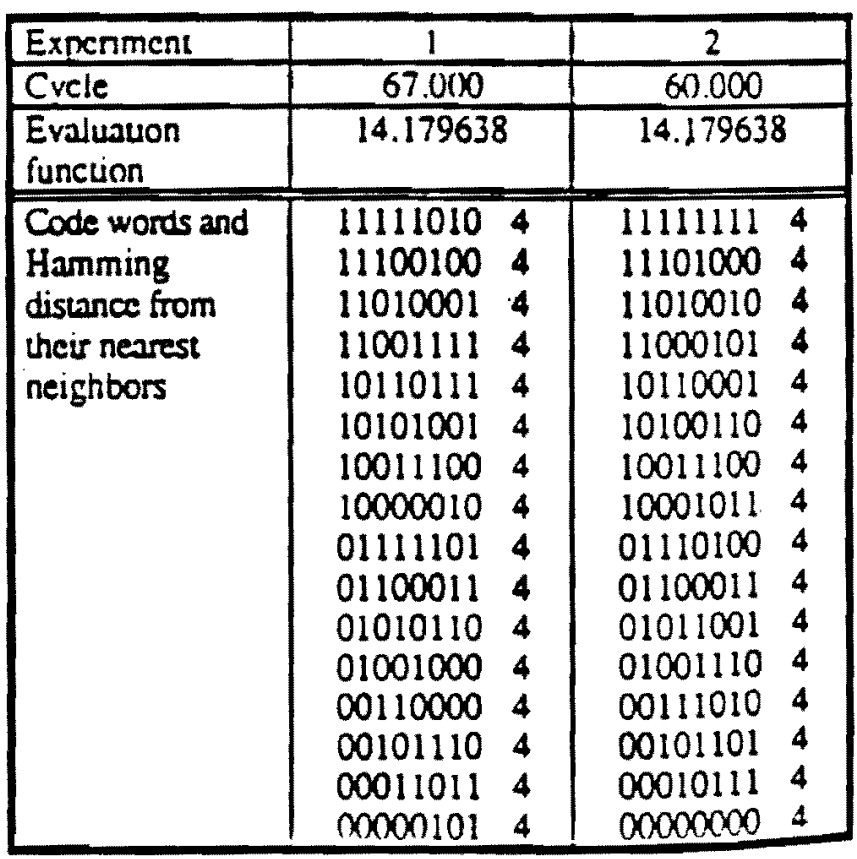

Figure 8: Solutions for the $(8,16,4)$ code.

This approach can also be extended to invesugate the usefulness of GAs for solving problems involving variable length source codes and cryplograpnic codes. 
cyclic codes, and non-block codes with estimated search spaces $2^{1000}$ large.

Another future direction would be to apply GAs at a higher level of concept and/or theory formacion. In the process of evaluating a large number of candidate codes. humans generally infer some general concepts, properies, or knowledge about the domain which can in turn be utilized to solve other similar problems more efficiently. For example, in coding theory there are numerous theorems which state (without having to perform uncerain search) whether a solution is possible to some problem. It would be highly desirable to be able to use GAS to discover, represent and then use this operational knowledge in order to build pracuical problem-solving procedures by these kinds of bootsuapping procedures.

\section{Acknowledgement}

This research was done in the Arificial Intelligence Research Center of George Mason University. Research activitues of the Center are suppored in par by the Office of Naval Researeh under grant No. N00014-88.K-0397. in par by the Orfice of Naval Research under grant No. NOOO1 14-88-K-0226, and in par by the Defense Advanced Research Projocts Agency under grant adminisicred by the Office of Naval Research, No. N00014.87-K.0874.

\section{References}

(1] Antonisse. J. (1989). A New Interpreusion of Schema Notation that Overturns the Binary Encoding Constrant. In Proceedings of the Third International Conference on Genetic Algorithms . pp. 86-91.

(2] De Jong, K. A.(1988), Learning with Genctic Algorithms: An Overview, Machine Learning Vol 3. pp. 121-138.

[2a) De Jong, K. A.(1975), An Analysis of the Bchavior of a Class of Gencuic Adaptuve Systems. (Doctoral Dissertation. University of Michiganl. Dissertation Abstracis Intemauonal. 36(10), 5i40B. (Universily Microtilms No. 76-9381).

[3] De Jong. K. A.(1990): Genetic Algonthms Based Leaming, Book Chapter in Kochine Liarning in Arminc:ai Intelligence Approach. Voiume 3, eds. Y. Koorstoif. R. S. Michalski.

[4] De Jong. K. A., and Spears. W. M. 1 1989). C'sing Genetc Algorithms to Sulve VP.Cumbietc Prodems. in Proceeainys of the Thira Internutwonus Cunierence on Geretue 14;0rtums . pp. 12\$-1:2

(5) Gumai. A. A.. Hemactandra, S. A. Shoerung, '., We: V.K. (1987), Using Simuialed innesinis:o
Design Good Codes, IEEE Transactions on Information Theory, TT-33, No 1, January 1987

[6] Garey, M. R. and Johnson, D. S.(1979). Compucers and Intractability: $A$ Guide to the Theory of NP. Completeness, W.H. Freeman and Company, San Fransisco, CA.

[7] Goldberg. D. E. (1989). Genetic Algorithms in Search Optimization and Machine learning. Addison Wesley Publishing Company, Inc.

[8] Grefenstette, I. (1984). GENESIS: A System for Using Genetic Search Procedures. In Proceedings of the 1984 Conjerence of Intelligent Systems and Machines. pp. 161-165.

[9] Grefenstette, J. (1986). Optimization of Control Parameters for Genetic Algorithms, IEEE Transactions on Systems. Man, and Cybernetics, 16, pp. 122-128.

[10] Hill, R. (1986), A First Course in Coding Theory. Oxford University Press, New York.

(11) Holland, J. H.(1975), Adaptation in Narural and Arificial Systems. The University of Michigan Press.

[12] Huffman. D. A. (1952), A Method for the Consuruction of Minimum Redundancy Codes. In Proc. IRE, vol 40, pp 1098-1101.

(13) Lin, S. and Costello, D. J., (1983), Error Control Coding: Fundamentais and Applications, Prenuce Hall NJ.

[14] Shannon, C. E. (1948), A Mathematical Theory of Communication, Bell System Tech. J., vol 27, (pt D). pp $379-423$ (pt II). pp 623-656.

[15] Sloane. N. J. A (1981), Recent Bounds for Codes. Sphere Packing and Related Problems Obtained by Linear Programming and other Methods. Contemporary Mathemancs 9, pp. 15j-185.

[16] Vitcrbi. A. J. and Omura. J. K. (1979). Principles of Digital Cummunicaton and Coding, McGraw Hill 